\title{
Stochastic modeling the effect of wind gust on dust entrainment during sand storm
}

\author{
CHENG XueLing, ZENG QingCun \& HU Fei \\ State Key Laboratory of Atmospheric Boundary Layer Physics and Atmospheric Chemistry, Institute of Atmospheric Physics, Chinese Academy of \\ Sciences, Beijing 100029, China
}

Received March 8, 2012; accepted April 17, 2012; published online May 30, 2012

\begin{abstract}
After the passage of a cold front, spring in northern China the outbreak of strong wind is often accompanied by dust emissions. Owing to the coherent structure of wind gust, dust particles can effectively overcome the systematic descending air motion and penetrate into the middle and upper levels of the atmospheric boundary layer, and then propagate further and diffuse into the troposphere where ascending air motion prevails. Here, we consider the coherent structure of wind gust in LS models, and construct a model suitable for the dust entrainment under the dust-storm canopy. With the parameter of gust, we simulate the dust entrainment during dust storms, and test that the coherent structures of wind gust make the sand particles to reach the upper of the boundary layer.
\end{abstract}

wind gust, coherent structure, dust entrainment, Lagrangian stochastic model

Citation: Cheng X L, Zeng Q C, Hu F. Stochastic modeling the effect of wind gust on dust entrainment during sand storm. Chin Sci Bull, 2012, 57: 3595-3602, doi: $10.1007 / \mathrm{s} 11434-012-5230-\mathrm{z}$

Sand storms and high concentrations of particle matter in the atmosphere may have important and sometimes devastating environmental impacts. One of the first researches to investigate the transport of sediment by wind was Bagnold [1]. His classical perception of the process served as a basis for any new developments, and it has been modified and sharpened at important points, in recent years. And now, there are two ways to simulate the process. One is Euler method. It uses two-phase flow model and regards particles as passive scalar $[2,3]$. But if the relaxation time of scalar is less than the turbulent flow Lagrangian timescale, which leads to the distinction between two speeds obvious such as the particle flow near the scalar source, or in the boundary layer, this model is not suitable.

Another is Lagrange method. This method follows every particle's random walk process. This is a Markov process, and Brownian motion is one type of this process, which is called Wiener type. There are three classical, but actually indistinct categories of particle motion determined by the

*Corresponding author (email: hufei@mail.iap.ac.cn) diameter of particles (Figure 1).

Larger (diameter bigger than $500 \mu \mathrm{m}$ ) or less exposed particles creep along the surface of the bed. They sometimes make small hops, and might eventually eject much smaller particles, contributing to the splash process. There have been few studies of creep and little is known about this type of motion. It is clear that the extreme difficulty of direct observation of particle movements close to and in the bed is the principal cause for this lack of information.

Aeolian saltation in its simplest manifestation is comprised of four linked process: aerodynamic entrainment, particle trajectories, particle-bed impacts (the splash problem) and wind field modification. Trajectories are modified parabolas, with initial angles close to $45^{\circ}$, small impacting angles, around $10^{\circ}$. The basic concept of such a model was developed earlier by Ungar and Haff [5], Werner [6]. Anderson and Haff [7], McEwan and Willetts [8] developed numerical models. Sorensen [9] gave solutions by analytical approximations. Recently, researchers continue to study the mechanisms of saltation $[10,11]$.

Dust particles are usually entrained into the air stream by 


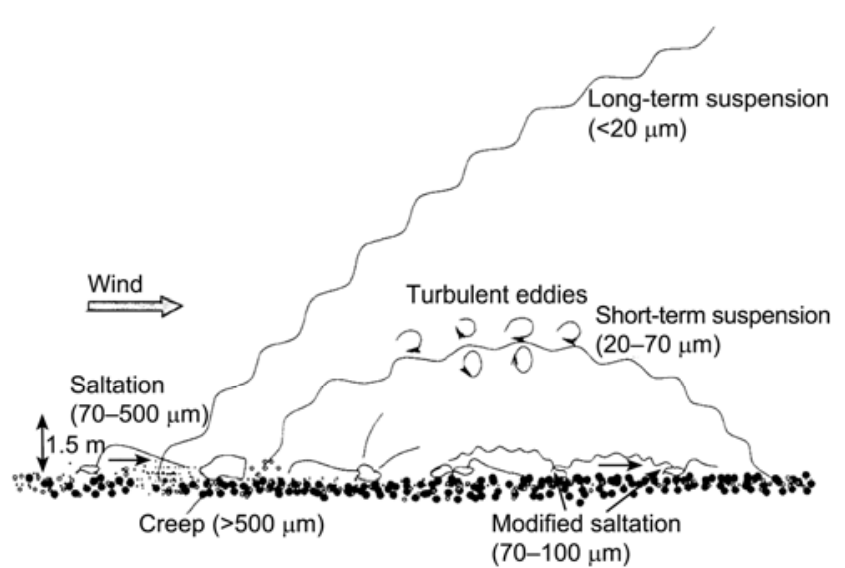

Figure 1 The motion and transport of dust particles [4].

the impact of saltating sand particles. Once entrained, dust particles enter suspension, and the fine particles are carried by turbulent eddies in the air stream and have the potential to be transported to great heights and over great distances. These particles have random trajectories, strongly influenced by the turbulent fluctuations of the flow. Many approaches exist for the calculation of dust heavy particle trajectories. The appeal of the random walk method is its directness, due to which it is relatively free of theoretical obscurity. Perhaps the most common heavy particle random walk model is the "eddy interaction model" $[12,13]$. This explicitly resolves particle acceleration, but treats the "driving" fluid velocity at the location of the particle as constant across "patches" of the fluid, and as changing discontinuously from eddy to eddy. So attentions are paid to model the stochastic particle velocity itself directly. It is usually by means of a Langevin-type equation, which will require to be provided the heavy particle velocity variance and the autocorrelation timescale along the trajectory [14]. Otherwise, there are also other methods to simulate the particle trajectory such as by the Ito-type equation $[15,16]$ or by the Monte Carlo model [17]. Wilson [18] investigated how well existing heavy particle trajectory models perform, relative to observations, and showed that the Langevin-type model suffices to obtain simulations that are about as accurate as we typically are accustomed to hope for in atmospheric modeling.

Wilson and Sawford [16] reviewed the application of Lagrangian stochastic (LS) models for the turbulent flow motion during passive tracer dispersion in turbulent atmospheric boundary layer flows. The "zeroth-order" LS model for motion along a single axis (the vertical), is a random walk in position (the drunkard's walk, or Random Displacement Model (RDM)). In the RDM, $\vec{y}_{i}$ is treated as Markovian, that is, correlation of particle velocity from one time step to the next is ignored; thus, the problem that the model is invalid for travel times short compared to the typical velocity correlation timescale. This deficiency is remedied in the "first-order" LS model, wherein the Markovian state variable is $\left(\boldsymbol{y}_{i}, \boldsymbol{V}_{i}\right)$, and the velocity evolves in time. The velocity can be solved using the equilibrium turbulent stochastic distribution $[19,20]$, or by turbulent model such as large-eddy simulation model to simulate the flow field [21], or by a generalized Langevin equation [22,23]. Thomson provided a constraint on the model coefficient, to ensure that the LS model has the property that, should it hypothetically be applied to the motion of tracer that is (already) well mixed in position-velocity space, the tracer would remain well mixed. A first-order LS model correctly predicts the rate of dispersion even in the near field of a source, where travel time is not larger than fluid-Lagrangian timescale, in contradistinction to the RDM and to Eulerian models. Of course, the trajectories are not valid for travel times on the order of the acceleration timescale. To treat such exceptionally short-range trajectories, or trajectories in low Reynolds number turbulence, one may introduce a second-order LS model [24]. In this case the Markovian state variable is $\left(\boldsymbol{y}_{i}, \boldsymbol{V}_{i}, \boldsymbol{A}_{i}\right)$, where $\boldsymbol{A}_{i}$ is the acceleration.

Despite this, the "first-order" LS model for turbulent dispersion in multidimensional flows remains problematic. This is because the well mixed condition is not sufficient to determine uniquely a model for turbulent dispersion in more than one dimension. This nonuniqueness is nontrivial since different models produce different predictions for the dispersion of tracers. Recently it has been shown that the nonuniqueness can be exploited to construct a model in "optimal" agreement with the measured dispersion of tracers. It is therefore demonstrated that such models are not universal, i.e. applicable to a wide range of flows without readjustment of model parameters. For example, predictions for scalar dispersion in the model plant canopy are obtained using the model of Flesch and Wilson [25], and the model of Reynolds [26].

In this paper, we will construct a model for the particles scalar dispersion in the dust-storm canopy with strong wind. After the passage of a cold front, spring in northern China the outbreak of strong wind is often accompanied by dust emissions [27]. During the period of strong winds, the soil erosion (sand and dust emission) is due to basic descending strong winds and fluctuations, and dust entrainment is mainly due to the coherent structure of wind gust [28-31]. Here, we will consider the coherent structure of wind gust in LS models, and construct a model suitable for the dust entrainment under the dust-storm canopy. From the analysis of the observation data, we got the parameterization of the wind gust. Here, we will use our model with the parameter of gust to simulate the process of dust entrainment and give a theoretical explanation on the dust entrainment by wind gust.

\section{The governing equation for heavy particle motion in a turbulent flows}

\subsection{Solid particle equation}

The equation governing the motion of a particle in a sta- 
tionary fluid is generally attributed to Basset (1888), Boussinesq (1903) and Oseen (1927). It is called the BBO equation. Moreover, the BBO equation was derived by assuming that the fluid flow near the particle could be split into two parts: the fluid flow without the particle and the flow disturbed by the presence of the particle. The forces acting on the particle were found by assuming that the disturbance flow can be treated as creeping flow and the inertia terms neglected. In a typical gas/particle flow, the ratio of the density of the fluid $\rho$ to the density of the particle $\rho_{\mathrm{p}}$ is small, and the motion of a heavy particle in an air flow is governed by the effects of viscous drag and gravity. Because the particle Reynolds number $R e_{\mathrm{p}}=\frac{d_{\mathrm{p}}|\boldsymbol{u}-\boldsymbol{V}|}{v}<<1$, here $d_{\mathrm{p}}$ is particle diameter, $\boldsymbol{V}$ is the particle velocity, $\boldsymbol{u}$ is the fluid velocity, $v$ is the kinematic viscosity, the viscous drag was limited to Stokes drag. Empirical relations can be used to account for the drag force on the particle, and we can write the equation of motion for a heavy particle as:

$$
\frac{\mathrm{d} \boldsymbol{V}(t)}{\mathrm{d} t}=\frac{[\boldsymbol{u}(\boldsymbol{y}(t), t)-\boldsymbol{V}(t)]}{\tau_{\mathrm{p}}} f-g \delta,
$$

where $\boldsymbol{y}$ is the particle coordinate, $\tau_{\mathrm{p}}$ is the particle relaxation time, assuming Stokes drag applies and is given by

$$
\tau_{\mathrm{p}}=\frac{\rho_{\mathrm{p}} d_{\mathrm{p}}^{2}}{18 \mu}
$$

The factor $f$ is the Stokes drag coefficient. $R e_{\mathrm{p}}<<1$, the flow is a Stokes flow and $f=1$. $R e_{\mathrm{p}} \geqslant 1, f$ becomes $f=1+$ $0.15 R e_{\mathrm{p}}^{0.687}$ [32].

Still, even in the simplest form of the grain movement equation, the biggest difficulty resides in the determination of the instantaneous fluid velocity along the particle trajectory $\boldsymbol{u}(\boldsymbol{y}(t), t)$. This is the velocity of the surrounding air at each position of the grain; it is the velocity of the fluid "seen" by the solid particle. Even though initially both the fluid and the solid particle are at the same position, because of the grain's inertia and the effects of gravity the two trajectories will be decorrelated, the velocities will be different and a relative velocity will appear. The solid particle will not remain within a single air parcel, but rather slips from one air parcel to another throughout the trajectory, probably moving out of the initial turbulent structure.

Next, we will estimate the fluid velocity "seen" by the solid particle by using a stochastic approach.

\subsection{Equation of fluid particle: the theory of Thomson}

The Lagrangian approach is a natural way of treating dispersion problems. Here, we will be interested in the case of a model with one particle and one time scale and we will consider that particles move independently from each other within the flow.

For large Reynolds numbers, $R e_{\mathrm{L}}=U L / v$ (which is the case in the atmosphere) and for time intervals greater than the Kolmogorov time scale $\left(\Delta t>>\tau_{\mathrm{K}}=\left(\frac{v}{\bar{\varepsilon}}\right)^{1 / 2}\right)$ the lagrangian autocorrelation timescale of acceleration, $T_{\gamma}$ is smaller than the lagrangian autocorrelation timescale of velocity, $T_{\mathrm{L}}$, as given by Monin and Yaglom [33]:

$$
\frac{T_{\gamma}}{T_{\mathrm{L}}} \approx \frac{1}{\sqrt{\operatorname{Re}_{\mathrm{L}}}}<<1 .
$$

Therefore, the velocity variations of a particle between 2 successive instants separated from $\Delta t$ are nearly independent. The velocity at $t+\Delta t$ depends only on the velocity $\boldsymbol{u}(t)$ and on the acceleration $\chi(t)$, it does not depend on other mechanisms acting on the acceleration for times smaller than $t$. With these considerations, the acceleration $\chi(t)$ can be modeled as a white noise and the couple of the position and velocity of the fluid element, $(\boldsymbol{x}, \boldsymbol{u})$ by a markovian process. It is of importance that the position alone is not a markovian process, since the autocorrelation timescale associated to the turbulent velocity is not negligible. Only the couple $(\boldsymbol{x}, \boldsymbol{u})$ is markovian.

If we chose randomly a fluid element, from all the fluid elements in the ensemble of flows, for $\Delta t>>\tau_{\mathrm{K}},(\boldsymbol{x}, \boldsymbol{u})$ will be a Markov process. The evolution of $(\boldsymbol{x}, \boldsymbol{u})$ can be described by the following, stochastic differential equations:

$$
\left\{\begin{array}{l}
\mathrm{d} x_{i}=u_{i} \mathrm{~d} t, \\
\mathrm{~d} u_{i}=a_{i}(\boldsymbol{x}, \boldsymbol{u}, t) \mathrm{d} t+b_{i j}(\boldsymbol{x}, \boldsymbol{u}, t) \mathrm{d} \xi_{j},
\end{array}\right.
$$

where the vector $a$ and the tensor $b$ are functions of $\boldsymbol{x}, \boldsymbol{u}$ and $t$, and $\mathrm{d} \xi$ are random increments of a Wiener process with independent components. The increments must be Gaussian with zero mean and variance $\mathrm{d} t$, and they have the following correlation property:

$$
<\mathrm{d} \xi_{i}(t) \mathrm{d} \xi_{j}(t+\tau)>=\delta_{i j}(t) \delta(\tau) \mathrm{d} t \mathrm{~d} \tau .
$$

If we consider the two-dimensional case of dispersion in a non-homogeneous turbulence the position and velocity of a fluid element are

$$
\boldsymbol{x}=\left\{\begin{array}{l}
x \\
0 \\
z
\end{array} \text { and } \tilde{\boldsymbol{u}}(\boldsymbol{x}, t)=\left\{\begin{array}{l}
U \\
0 \\
w(z, t)
\end{array} .\right.\right.
$$

The general form of a two-dimensional differential stochastic equation is:

$$
\left\{\begin{array}{l}
\mathrm{d} x=U \mathrm{~d} t \\
\mathrm{~d} w=a(z, w, t) \mathrm{d} t+b(z, w, t) \mathrm{d} \xi \\
\mathrm{d} z=w \mathrm{~d} t .
\end{array}\right.
$$

In two-dimensional homogeneous isotropic turbulence, 
the stochastic system becomes

$$
\left\{\begin{array}{l}
\mathrm{d} x=U \mathrm{~d} t \\
\mathrm{~d} z=w \mathrm{~d} t, \\
\mathrm{~d} w=-\frac{C_{0}}{2} \frac{\bar{\varepsilon}}{\sigma_{w}^{2}} w \mathrm{~d} t+\sqrt{C_{0} \bar{\varepsilon}} \mathrm{d} \zeta,
\end{array}\right.
$$

where $\bar{\varepsilon}$ is the ensemble average rate of dissipation of turbulent energy and $C_{0}$ is the Kolmogorov constant [34], experimentally estimated to be $2.1, \sigma_{w}$ is the standard deviation of the vertical velocity distribution.

\subsection{Modeling of the dispersion of heavy particles in a turbulent boundary layer flow}

Originally, the solid particle and the fluid particle are at the same location $A$ in the same turbulent eddy (Figure 2). At the next time step $\Delta t$, the fluid particle moves to $B$ and the solid particle to $C$, due to gravity and inertia. The solid particle is now surrounded by a new fluid particle. A decorrelation occurs at every time step and we will note $\Delta r$ the separation between the particle and the fluid element which at previous time was the driving fluid element. Provided the separation between the fluid and the solid particles does not exceed the fluid integral length scale $L$, we could consider the driving fluid velocity in $C$ to be correlated with its previous value. Otherwise, the solid particle has moved into another turbulent structure.

The particles are lifted up in different ways depending on the diameter, density ratio, trajectories of the fluid and particles, kinetic energies and inertia of the particles and the fluid. The vortices sweep along the ground, pushing particles out of the way. In doing so, they create conditions where particles may be entrained and lifted up if they are light enough. For example, one option is that the particle may be simply lifted up with the burst all the way to the outer region. Another option is that the particle is tossed sideways and/or upward, away from the vortex. Also, a third possibility is that the particle is lifted up and comes back towards the ground and then lifted up again. In these cases, the turbulent structures are the primary entrainment mechanism and that the ejection depends on the characteristics of

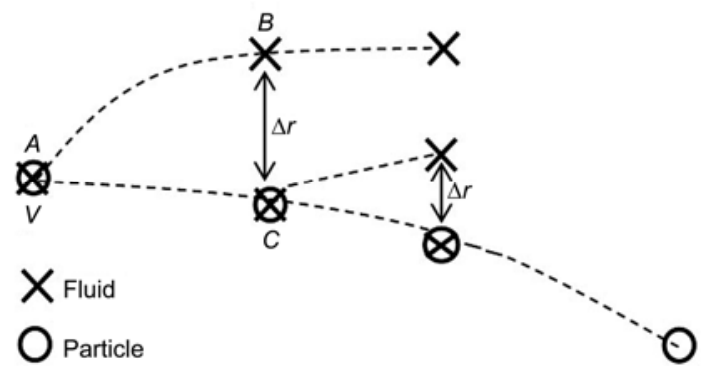

Figure 2 The different velocities between the solid particle and the air parcel initially surrounded the particle. the particles. But according to recent analysis of observation data during strong wind period after the passage of cold wave in spring in northern China, the soil erosion (sand and dust emission) is due to basic descending strong winds and fluctuations, and dust entrainment is mainly due to wind gust. When considering a strong wind with descending air flow over the ground surface, the particles are injected into the flow by the turbulent structures and basic flow [27,28]. The transport of particles consists of the computations of the following equations: (1) Lagrangian equation of the motion of the solid particle; (2) Stochastic equations for the fluid velocity along the fluid particle trajectory; and (3) Modified stochastic equations for the fluid velocity along the solid particle trajectory.

\section{The effect of wind gust in the sand-dust entrainment in the real atmosphere}

In East Asia, especially in northern China and Mongolia, the large scale sand blowing and dust-storm weather events almost occur in spring and all are accompanied with strong wind after the passage of cold front. By analyzing the data of atmospheric boundary layer during every such weather, it shows that the characteristics of wind gust and other quantities such as turbulent energy, friction velocity and many others can be very well expressed by parameterization formulas [28,29,31]. Here, we use these formulas to give the initial and boundary conditions to calculate the sand-dust entrainment in the real atmospheric boundary layer.

Because there are strong wind shears and pulses of wind and temperature in the atmospheric boundary during the strong wind period, the strong vertical mixes make the stratification of the boundary layer nearly neutral. The air flow is approximately considered as the quasi-two dimensional incompressible flow.

The wind profile characteristics and the turbulent characteristics of the flow are get from the observation data $[28,29,31]$, they are

$$
\begin{aligned}
& \begin{cases}u(z)=u_{\text {sur }}+u_{0}\left(\frac{z}{\delta}\right)^{0.277}, & z \leqslant \delta, \\
u=13 \mathrm{~m} / \mathrm{s}, & z>\delta, \\
u_{\text {sur }}=1 \mathrm{~m} / \mathrm{s}, & u_{0}=12 \mathrm{~m} / \mathrm{s}, \\
\delta=240 \mathrm{~m} .\end{cases} \\
& u_{t^{*}}(z, S)=\alpha_{0}(z)-\alpha_{1}(z) \mathrm{e}^{-0.15 s}, \\
& \quad \alpha_{0}(z)=\left(0.93+\frac{z}{30}\right) \mathrm{e}^{-0.228\left(0.93+\frac{z}{30}\right)}, \\
& \quad \alpha_{1}(z)=\left(0.84+\frac{z}{35}\right) \mathrm{e}^{-0.257\left(0.84+\frac{z}{35}\right)} ;
\end{aligned}
$$




$$
\begin{aligned}
\sigma_{w}(z, S) & =\beta_{0}(z)-\beta_{1}(z) \mathrm{e}^{-0.15 S}, \\
\beta_{0}(z) & =\left(1.46+\frac{z}{25}\right) \mathrm{e}^{-0.143\left(1.46+\frac{z}{25}\right)}, \\
\beta_{1}(z) & =\left(1.11+\frac{z}{35}\right) \mathrm{e}^{-0.193\left(1.11+\frac{z}{35}\right)}
\end{aligned}
$$

where $S=h^{*}\left(\frac{\partial \bar{u}}{\partial z}\right) \approx u_{t^{*}-120 \mathrm{~m}} \cdot h^{*} \frac{\bar{u}_{280 \mathrm{~m}}-\bar{u}_{120 \mathrm{~m}}}{280 \mathrm{~m}-120 \mathrm{~m}}$ is the controlling factor for the turbulent characteristics, $u_{t^{*}-120 \mathrm{~m}}$ is the turbulent friction velocity at $120 \mathrm{~m}$ height, $h^{*}$ is taken as the thickness of descending sub layer $(\bar{w} \leqslant 0)$ in our case [31].

The following relations for the turbulent characteristics of the flow were used:

Turbulent kinetic energy dissipation: $\bar{\varepsilon}=\frac{5}{C_{\mathrm{o}}} \frac{u_{t^{*}} \sigma_{w}{ }^{2}}{z}$.

The Lagrangian correlation timescale: $T_{\mathrm{L}}=\frac{2}{C_{\mathrm{o}}} \frac{\sigma_{w}{ }^{2}}{\bar{\varepsilon}}$, $C_{\mathrm{o}}=2.1$.

The time step $\Delta t=\min \left(\tau_{\mathrm{p}}, 0.05 T_{\mathrm{L}}\right)$. The density of particle is: $\rho_{\mathrm{p}}=1500 \mathrm{~kg} / \mathrm{m}^{3}$ (dust), $\rho_{\mathrm{p}}=1700 \mathrm{~kg} / \mathrm{m}^{3}$ (dust and sand) and $\rho_{\mathrm{p}}=2650 \mathrm{~kg} / \mathrm{m}^{3}$ (sand). The particle source position lies in the first $100 \mathrm{~m}$ length of the entire ground, and the position is Gaussian with mean of $50 \mathrm{~m}$ and variance of $50 \mathrm{~m}$, and near the groud $z=0.002 \mathrm{~m}$. Particles are injected into the flow randomly over the entire ground. The injection angle and vertical velocity are random variables with Gaussian Probability Density Functions. The mean and the standard deviation are [27]:

$$
\left\{\begin{array}{l}
<V_{2}>=2 u_{t^{*}{ }_{-} \text {sur }}=1.2 \mathrm{~m} / \mathrm{s}, \quad \sigma_{V_{2}}=\frac{u_{t^{*} \_ \text {sur }}}{2}=0.3 \mathrm{~m} / \mathrm{s}, \\
<\alpha>=45^{\circ}, \quad \sigma_{\alpha}=45^{\circ}
\end{array}\right.
$$

where $u_{t^{*} \text { sur }}$ is the turbulence friction velocity near the surface.

The particles are transported in suspension and they do not have enough energy to eject new particles when they impact the bed. Therefore no ejection is considered here. And when the particles impact the ground, their rebound is not considered. In order to test the capabilities of dispersion, the trajectories of particles were computed over $5000 \mathrm{~m}$. The particles' diameter are 5, 20 and $40 \mu \mathrm{m}$, respectively.

First, we simulated the sand-dust entrainment with horizontal basic flow. In the Figure 3, there are 50 particles in each suspension. It is indicated by the theoretical analysis that the thickness of sand/dust two phase flows in the boundary layer of the air is only about $1.5 \mathrm{~m}$. And very few of them can reach $150 \mathrm{~m}$ height, i.e. 100 times of the thickness of the two phase sand/dust fluid flow [28,29]. The simulation results show that there are $34 \%$ dust particles,
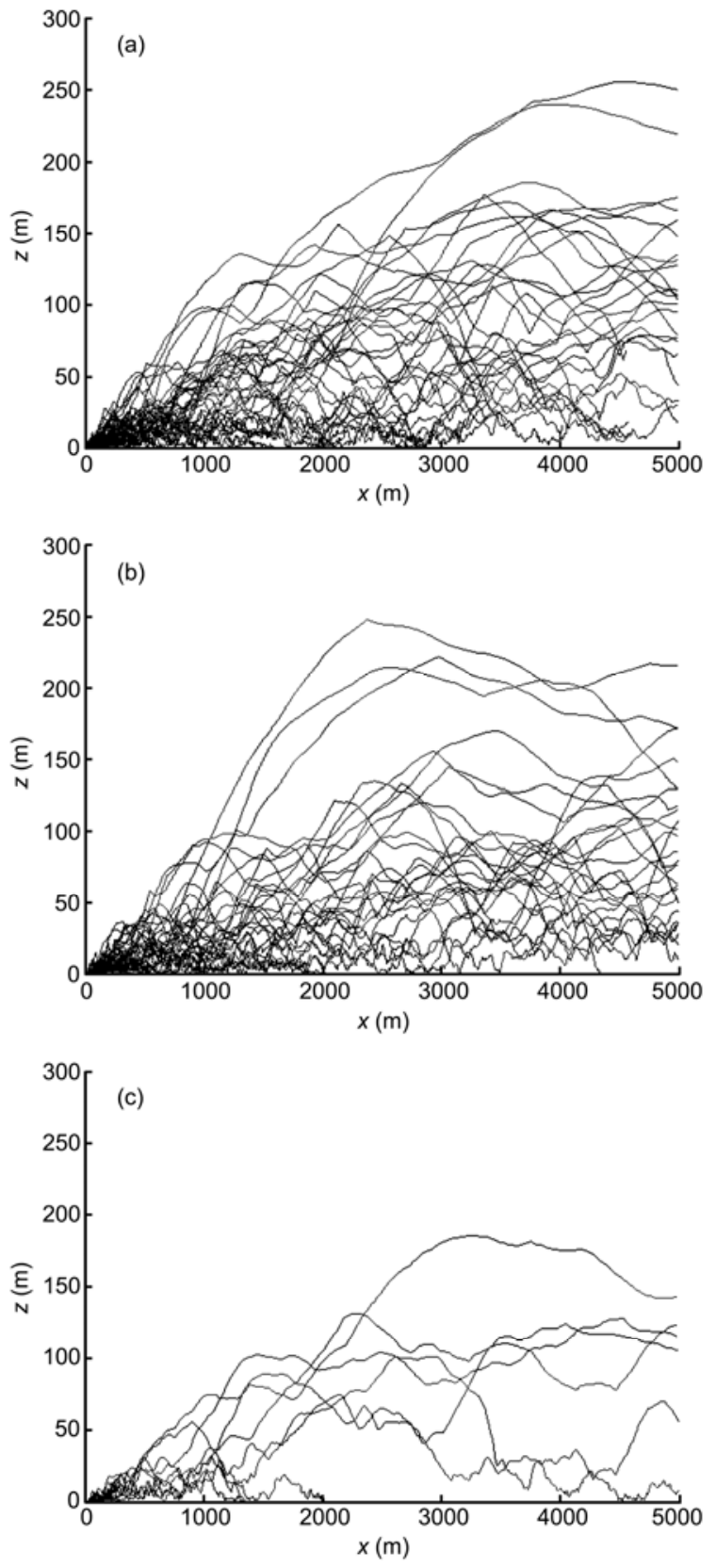

Figure 3 Simulation of dust entrainment process without wind gust and descending flow. (a) Dust $\left(\rho_{\mathrm{p}}=1500 \mathrm{~kg} / \mathrm{m}^{3}, d_{\mathrm{p}}=5 \mu \mathrm{m}\right)$; (b) dust and sand $\left(\rho_{\mathrm{p}}=1700 \mathrm{~kg} / \mathrm{m}^{3}, d_{\mathrm{p}}=20 \mu \mathrm{m}\right) ;(\mathrm{c})$ sand $\left(\rho_{\mathrm{p}}=2650 \mathrm{~kg} / \mathrm{m}^{3}, d_{\mathrm{p}}=40 \mu \mathrm{m}\right)$.

$48 \%$ dust and sand particles and $88 \%$ sand particles in the $1.5 \mathrm{~m}$ height layer. The most height where the dust or dust and sand particles can reach is about $250 \mathrm{~m}$, and $180 \mathrm{~m}$ for sand, where is still the lower layer of the atmospheric boundary.

Next, considering a strong wind with descending air flow over the ground surface, the particles are injected into the flow by the turbulent structures and basic flow [27-29]. Then we simulated the sand-dust entrainment with horizontal basic flow and descending basic flow. The horizontal wind profile is the same with eq. (9), and the vertical wind 
profile is

$$
\bar{w}=-0.0033+1.34 \times 10^{-5} z^{2} .
$$

The friction velocity, the standard deviation of vertical velocity, the turbulent kinetic energy dissipation and the Lagrangian correlation timescale are all same with first example. And the particles are also the same.

In Figure 4, there are 50 particles in each suspension. The simulation results show that, in this situation, there are 92\% dust particles, $72 \%$ dust and sand particles and $98 \%$ sand particles in the $1.5 \mathrm{~m}$ height layer. The most height where the dust particles can reach is about $20 \mathrm{~m}$, only one dust/sand particle can reach $50 \mathrm{~m}$ height and others are below $30 \mathrm{~m}$, and also one sand particle reach $50 \mathrm{~m}$ height, others can reach no more than $1 \mathrm{~m}$.

Third, the wind gust is introduced. For the equivalent horizontal amplitude $A_{\mathrm{gh}}$ and the equivalent vertical amplitude $A_{\mathrm{gw}}$ of the wind gust change with height [31],

$$
\begin{aligned}
A_{\mathrm{gh}}(z, G) & =a_{0}(z)-a_{1}(z) \mathrm{e}^{-0.38 G}, \\
a_{0}(z) & =\left(1.72+\frac{z}{25}\right) \mathrm{e}^{-0.136\left(1.72+\frac{z}{25}\right)}, \\
a_{1}(z) & =\left(1.17+\frac{z}{50}\right) \mathrm{e}^{-0.245\left(1.17+\frac{z}{50}\right)},
\end{aligned}
$$
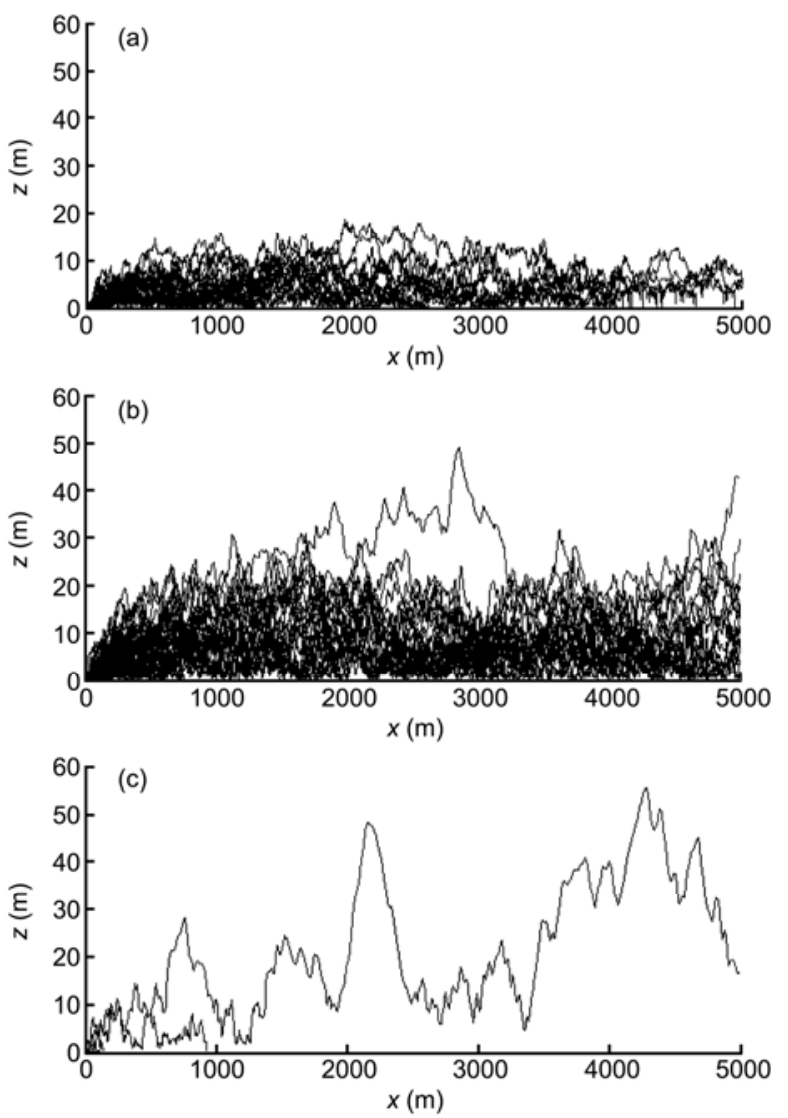

Figure 4 Simulation of dust entrainment process with descending flow. (a) Dust $\left(\rho_{\mathrm{p}}=1500 \mathrm{~kg} / \mathrm{m}^{3}, d_{\mathrm{p}}=5 \mu \mathrm{m}\right)$; (b) dust and sand $\left(\rho_{\mathrm{p}}=1700 \mathrm{~kg} / \mathrm{m}^{3}\right.$, $\left.d_{\mathrm{p}}=20 \mu \mathrm{m}\right)$, (c) sand $\left(\rho_{\mathrm{p}}=2650 \mathrm{~kg} / \mathrm{m}^{3}, d_{\mathrm{p}}=40 \mu \mathrm{m}\right)$.

$$
\begin{aligned}
A_{\mathrm{gw}}(z, G) & =b_{0}(z)-b_{1}(z) \mathrm{e}^{-0.38 G}, \\
b_{0}(z) & =\left(0.2+\frac{z}{60}\right) \mathrm{e}^{-0.37\left(0.2+\frac{z}{60}\right)}, \\
b_{1}(z) & =\left(0.09+\frac{z}{100}\right) \mathrm{e}^{-0.98\left(0.09+\frac{z}{100}\right)},
\end{aligned}
$$

where $G$ is the controlling factor for the gusty wind characteristics whose dimension is energy, $G \approx u_{t^{*}-120 \mathrm{~m}} \cdot S$.

The wind gust disturbances $\left(u_{g}, w_{g}\right)$ are given as

$$
\begin{aligned}
& u_{\mathrm{g}}=A_{\mathrm{gh}} \cos \left[\theta_{\mathrm{gh}}+\frac{2 \pi}{T_{\mathrm{g}}}\left(t-\frac{z}{c_{\perp}}\right)\right], \\
& w_{\mathrm{g}}=A_{\mathrm{gw}} \sin \left[\theta_{\mathrm{gw}}+\frac{2 \pi}{T_{\mathrm{g}}}\left(t-\frac{z}{c_{\perp}}\right)\right],
\end{aligned}
$$

where $\theta_{\mathrm{gh}}$ and $\theta_{\mathrm{gw}}$ are the initial horizontal and vertical phase angle of wind gust, here are zero. $T_{\mathrm{g}}$ is the equivalent period of gusty train, $g T_{\mathrm{g}} \approx c_{0}-c_{1} \mathrm{e}^{-0.25 S}=34.2-56.4 \mathrm{e}^{-0.25 S}$, whose unit is $\operatorname{min~} \mathrm{m} / \mathrm{s}^{2}$. And $c_{\perp}=1 \mathrm{~m} / \mathrm{s}$ is the downward speed of gust wind.

And Figure 5 is the particle trajectories under the wind gust. For the coherent structures of wind gust, the particles
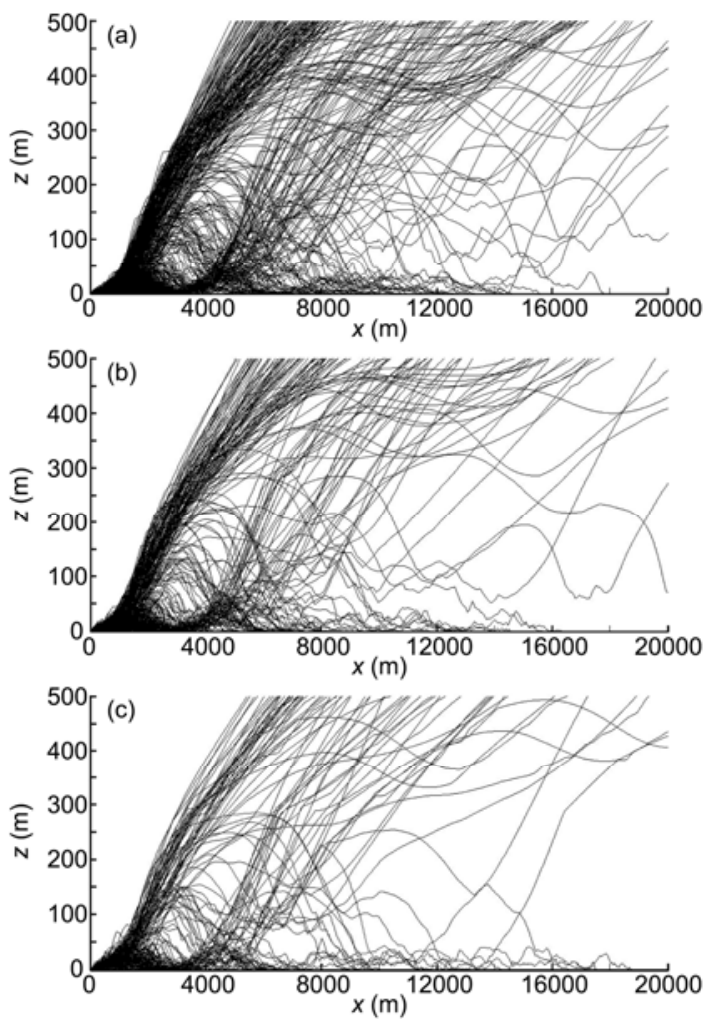

Figure 5 Simulation of dust entrainment process with wind gust. (a) Dust $\left(\rho_{\mathrm{p}}=1500 \mathrm{~kg} / \mathrm{m}^{3}, d_{\mathrm{p}}=5 \mu \mathrm{m}\right) ;\left(\right.$ b) dust and sand $\left(\rho_{\mathrm{p}}=1700 \mathrm{~kg} / \mathrm{m}^{3}, d_{\mathrm{p}}=20 \mu \mathrm{m}\right)$; (c) sand $\left(\rho_{\mathrm{p}}=2650 \mathrm{~kg} / \mathrm{m}^{3}, d_{\mathrm{p}}=40 \mu \mathrm{m}\right)$. 
can reach the top of the atmospheric boundary layer. But as the particle is heavier, the numbers which can entrain into higher level are less.

Figure 6 is the particle trajectories under the real wind condition, comparing to Figure 5, the particle trajectories look alike. It tests that the parameterizations of wind gust are reasonable. And in the model, we need consider the wind gust and can use the parameterizations of them to calculate the dust flux.

Figure 7 is the numbers of different particles reaching
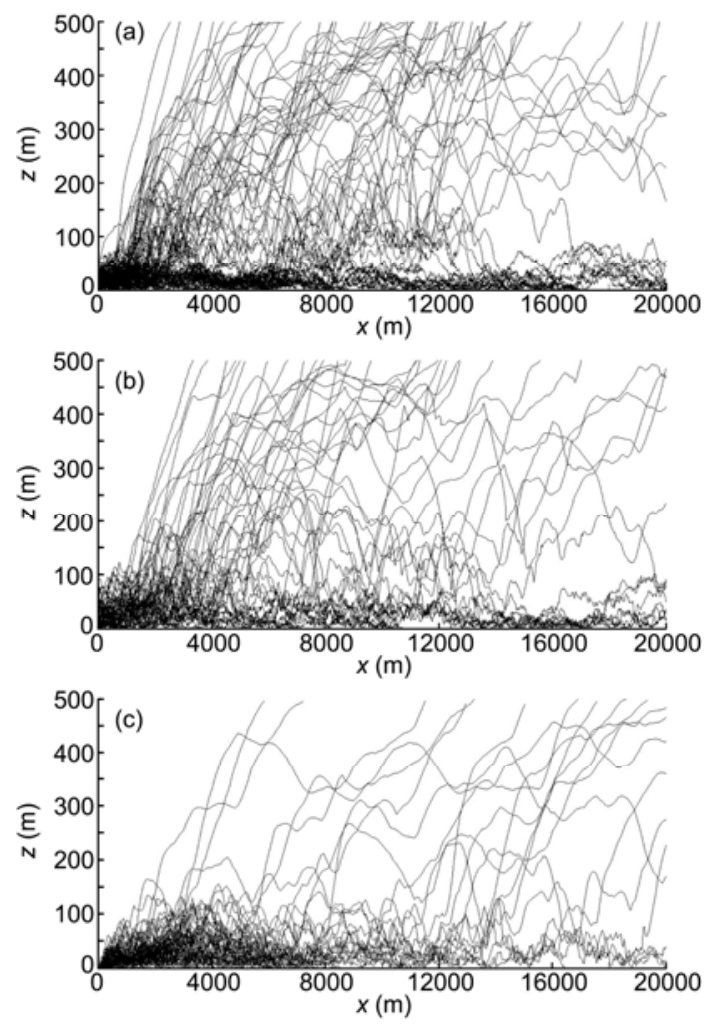

Figure 6 The dust entrainment process with real wind speed. (a) Dust $\left(\rho_{\mathrm{p}}=1500 \mathrm{~kg} / \mathrm{m}^{3}, d_{\mathrm{p}}=5 \mu \mathrm{m}\right) ;\left(\right.$ b) dust and sand $\left(\rho_{\mathrm{p}}=1700 \mathrm{~kg} / \mathrm{m}^{3}, d_{\mathrm{p}}=20 \mu \mathrm{m}\right)$; (c) sand $\left(\rho_{\mathrm{p}}=2650 \mathrm{~kg} / \mathrm{m}^{3}, d_{\mathrm{p}}=40 \mu \mathrm{m}\right)$.

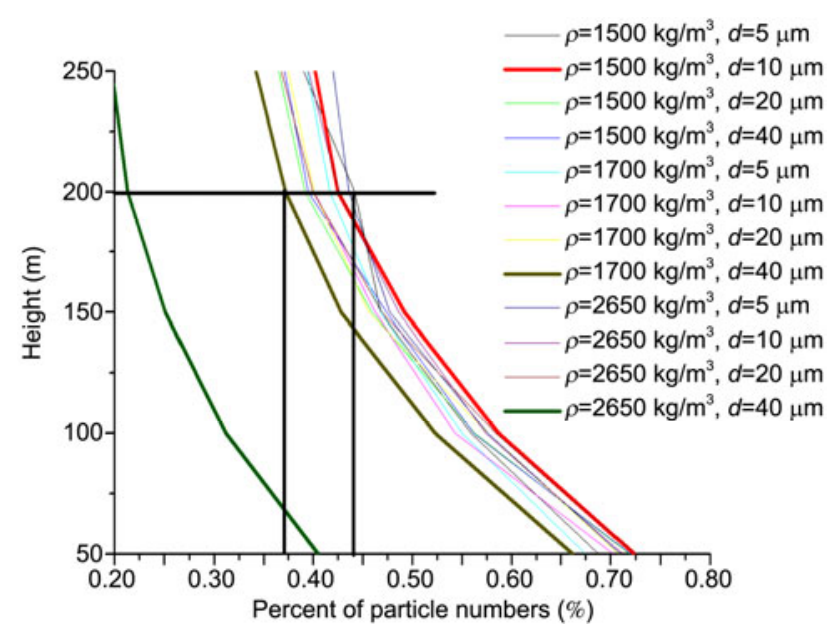

Figure 7 The particle numbers on various heights. various heights. From the picture, we can see that expect the heaviest particles, about $37 \%-43 \%$ particles can reach the $200 \mathrm{~m}$ height.

\section{Conclusion}

There is often an outbreak of strong winds following the passage of a spring cold front in East Asia. The winds burst very suddenly, and with gustiness. The wind gust is characterized by a coherent structure. During the strong basic winds, there is systematic downward motion at the lower levels of the atmospheric boundary layer, the analysis in this paper has shown, that during strong winds, the systematic strong descending air motion suppresses the sand/dust particles and they cannot move up into the middle and upper levels of the atmospheric boundary layer, instead accumulating in the very low levels near the ground surface. Only due to the coherent structure of the wind gust, the sand/dust particles have $d \approx 5-40 \mu \mathrm{m}$, can entrainment into the middle and upper levels of the atmospheric boundary layer and the troposphere occur. So, dust particles entrainment is in an impulsive form one group after another. This is the typical picture of soil erosion and sand/dust emission, as well as dust entrainment processes during spring in eastern Asia.

This work was supported by the National Natural Science Foundation of China (40830103 and 40875008), and the National Basic Research Program of China (2010CB951804).

1 Bagnold R A. The Physics of Blown Sand and Desert Dunes. New York: Chapman and Hall, 1941. 265

2 Friedlander S K, Johnstone H F. Deposition of suspended particles from turbulent gas streams. Ind Eng Chem, 1957, 49: 1151

3 Young J, Leeming A. A theory of particle deposition in turbulent pipe flow. J Fluid Mech, 1997, 340: 129

4 Shao Y P. Physics and Modeling of Wind Erosion. Dordrecht/Boston/ London: Kluwer Academic Publishers, 2000. 393

5 Ungar J E, Haff P K. Steady state saltation in air. Sedimentology, 1987, 34: 289-299

6 Werner B T. A steady-state model of wind-blown sand transport. J Geol, 1990, 98: 1-17

7 Anderson R S, Haff P K. Wind modification and bed response during saltation of sand in air. Acta Mech, 1991, (Suppl. 1): 21-51

8 McEwan I K, Willetts B B. Numerical model of the saltation cloud. Acta Mech, 1991, (suppl 1): 53-66

9 Sorensen M. An analytic model of wind-blown sand transport. Acta Mech, 1991, (suppl 1): 67-81

10 Shao Y, Li A. Numerical modelling of Saltation in the atmospheric surface layer. Boundary-Layer Meteorol, 1999, 91: 199-225

11 Zou X Y, Wang Z L, Hao Q Z, et al. The distribution of velocity and energy of saltating sand grains in a wind tunnel. Geomorphology, 2001, 36: 155-165

12 Kallio G A, Reeks M W. A numerical simulation of particle deposition in turbulent boundary layers. Int J Multiph Flow, 1989, 15: 433

13 Graham D I. An improved eddy interaction model for numerical simulation of turbulent particle dispersion. J Fluids Eng (Trans ASME), 1996, 118: 819-823

14 Walklate P J. A random-walk model for dispersion of heavy particles in turbulent flow. Boundary-Layer Meteorol, 1987, 39: 175-190 
15 Shao Y P. A Lagrangian Stochastic Model for nonpassive particle diffusion in turbulent flows. Mathl Comput Model, 1995, 21: 31-37

16 Wilson J D, Sawford B L. Review of Lagrangian stochastic models for trajectories in the turbulent atmosphere. Boundary-Layer Meteorol, 1996, 78: 191-210

17 Turfus C, Hunt J C R. A stochastic analysis of the displacements of fluid elements in inhomogeneous turbulence using Kraichnan's method of random modes. In: Comte-Bellot G, Mathie J, eds. Advances in Turbulence. Berlin: Springer-Verlag, 1987. 191-203

18 Wilson J D. Trajectory models for heavy particles in atmospheric turbulence: Comparison with observations. J Appl Meteorol, 2000, 39: 1894

19 Edson J B, Fairall C W. Spray droplet modeling. I: Lagrangian model simulation of the turbulent transport of evaporating droplets. J Geophys Res, 1994, 99: 25295-25311

20 Zheng X J, Wang P. Numerical simulation on stochastic movement of sands in wind-blown sand (in Chinese). J Desert Res, 2006, 26: 184-188

21 Cai X H, Zhang R, Li Y. A Large-eddy simulation and Lagrangian stochastic study of heavy particle dispersion in the convective boundary layer. Boundary-Layer Meteorol, 2006, 120: 413-435

22 Thomson D J. Criteria for the selection of stochastic models of particle trajectories in turbulent flows. J Fluid Mech, 1987, 180: 529-556

23 Guo Y, Cui G X, Xu C X, et al. A stochastic model of heavy particle dispersion in turbulent boundary layer (in Chinese). Chin J Comput Phys, 2004, 21: 515-522

24 Du S, Sawford B L, Wilson J D, et al. A determination of the Kolmogorov constant $\left(C_{0}\right)$ for the Lagrangian velocity structure function, using a second-order Lagrangian stochastic model for decaying ho- mogeneous, isotropic turbulence. Phys Fluids, 1995, 7: 3083-3090

25 Flesch T K, Wilson J D. A two dimensional trajectory simulation model for nongaussian, inhomogeneous turbulence within plant canopies. Boundary Layer Meteorol, 1992, 61: 349-374

26 Reynolds A M. On the formulation of Lagrangian stochastic models of scalar dispersion within plant canopies. Boundary-Layer Meteorol, 1998, 86: 333-344

27 Zeng Q C, Dong C H, Peng G B, et al. Gigantic Yellow Cloud-The Dust Storm in Eastern Asia (in Chinese). Beijing: Science Press, 2006

28 Zeng Q C, Cheng X L, Hu F, et al. Gustiness and coherent structure of strong wind and their role in the dust emission and entrainment. Adv Atmos Sci, 2010, 27: 1-13

29 Cheng X L, Zeng Q C, Hu F. Characteristics of gusty wind disturbances and turbulent fluctuations in windy atmospheric boundary layer behind cold fronts. J Geophys Res, 2011, 116: D06101

30 Cheng X L, Hu F, Zeng Q C. The simulation of wind gust structure in atmospheric boundary layer with lattice Boltzmann method. Chin Sci Bull, 2012, 57, doi: 10.1007/s11434-011-4950-9

31 Cheng X L, Zeng Q C, Hu F. Parameterizations of some important characteristics of turbulences and gusts in the atmospheric boundary layer. J Geophys Res, 2012, 117: D08113

32 Clift R, Grace J R, Weber M E. Bubbles, Drops and Particles. New York: Academic Press, 1978

33 Monin A S, Yaglom A M. Statistical Fluid Mechanics. Cambridge: M.I.T. Press, 1975. 605

34 Cheng X L, Wang B L, Hu F, et al. Kolmogorov constants of atmospheric turbulence over a homogeneous surface. Atmos Oceanic Sci Lett, 2010, 3: 195-200

Open Access This article is distributed under the terms of the Creative Commons Attribution License which permits any use, distribution, and reproduction in any medium, provided the original author(s) and source are credited 